(2) Open Access Full Text Article

REVIEW

\title{
In-vitro blood-brain barrier models for drug screening and permeation studies: an overview
}

This article was published in the following Dove Press journal:

Drug Design, Development and Therapy

\author{
Sounak Bagchi ${ }^{1}$ \\ Tanya Chhibber' \\ Behnaz Lahooti' \\ Angela Verma' \\ Vivek Borse ${ }^{2}$ \\ Rahul Dev Jayant ${ }^{\prime}$ \\ 'Department of Pharmaceutical Sciences, \\ School of Pharmacy, Texas Tech \\ University Health Sciences Center, \\ Amarillo, TX 79I06, USA; ${ }^{2}$ Centre for \\ Nanotechnology, Indian Institute of \\ Technology Guwahati, Guwahati, Assam \\ 781039, India
}

\begin{abstract}
The blood-brain barrier (BBB) is comprised of brain microvascular endothelial central nervous system (CNS) cells, which communicate with other CNS cells (astrocytes, pericytes) and behave according to the state of the CNS, by responding against pathological environments and modulating disease progression. The BBB plays a crucial role in maintaining homeostasis in the CNS by maintaining restricted transport of toxic or harmful molecules, transport of nutrients, and removal of metabolites from the brain. Neurological disorders, such as NeuroHIV, cerebral stroke, brain tumors, and other neurodegenerative diseases increase the permeability of the BBB. While on the other hand, semipermeable nature of $\mathrm{BBB}$ restricts the movement of bigger molecules i.e. drugs or proteins $(>500 \mathrm{kDa})$ across it, leading to minimal bioavailability of drugs in the CNS. This poses the most significant shortcoming in the development of therapeutics for CNS neurodegenerative disorders. Although the complexity of the BBB (dynamic and adaptable barrier) affects approaches of CNS drug delivery and promotes disease progression, understanding the composition and functions of BBB provides a platform for novel innovative approaches towards drug delivery to CNS. The methodical and scientific interests in the physiology and pathology of the BBB led to the development and the advancement of numerous in vitro models of the BBB. This review discusses the fundamentals of BBB structure, permeation mechanisms, an overview of all the different in-vitro BBB models with their advantages and disadvantages, and rationale of selecting penetration prediction methods towards the critical role in the development of the CNS therapeutics.
\end{abstract}

Keywords: blood-brain barrier, BBB, brain microvascular endothelial cells, BMECs, tight junctions, TJs, proteins, central nervous system, CNS, induced pluripotent cells, iPSCs, insilico prediction methods

\section{Introduction}

The first evidence for the existence of a barrier between the central nervous system (CNS) and the systemic circulation was reported by Paul Ehrlich in 1885 and Edwin Goldmann in 1913 and the term "Blood-brain barrier" (BBB) was coined by Stern and Gaultier in 1922. ${ }^{1,2}$ The nature of the BBB is semipermeable as it restricts the movement of detrimental molecules and cells from the blood and allows uptake of selective nutrients and hormones. The major cells comprising the BBB are brain microvascular endothelial cells (BMECs), which are supported by astrocytes, and pericytes. ${ }^{3}$ We briefly introduce the biological properties and functions of individual BBB components to discuss in vitro BBB models. ${ }^{1,4,5}$ The assurance of the access of nutrients to the brain is due to the large surface area, and the diminutive diffusion distance from the $\mathrm{BBB}$ capillaries to the neurons in the
Correspondence: Rahul Dev Jayant Department of Pharmaceutical Sciences, School of Pharmacy, Texas Tech University Health Sciences Center, 1406 S. Coulter Street, Suite II04, Amarillo, TX 79106, USA

Tel +18064149154

Fax +I 8063564034

Email rd.jayant@ttuhsc.edu 
CNS and the chemicals/molecules penetrate the BBB by utilizing intra- and intercellular routes. Tight junctions (TJs) to facilitate the passage of molecules based on their lipophilicity, ionization, polarity and other physicochemical properties regulate the intracellular route whereas, the intercellular route [(transport of molecules from the luminal (apical) to the abluminal (basolateral) side of endothelial cells] is controlled by passive diffusion, endocytosis, and the ratio of influx and efflux transporters. ${ }^{6}$

The inefficacy of drugs to reach across the BBB is mainly due to poor physico-chemical or pharmacokinetic properties, i.e. inefficient absorption, distribution, metabolism, and excretion (ADME). In addition to ADME, the toxicity of CNS drugs is also one amongst the significant shortcomings. ${ }^{8}$ Drug exposure is controlled by plasma pharmacokinetic properties of the drug, which are different from the brain pharmacokinetics of the drug. Studying drug pharmacokinetics for CNS specifically involves understanding the correlation of physicochemical properties of drug compound and physiological function of the BBB. ${ }^{1,6,8}$ Therefore, in this review, we have discussed the fundamentals of BBB focusing on the permeation mechanisms, penetration measurement, prediction methods and diseases patterns that have been changing in recent times. In the case of infectious diseases of CNS or ageing disorders, the most significant factor is the incapability of BBB to maintain its integrity and open temporarily allowing the access of the drugs into the CNS. Unable to maintain the brain homeostasis and allowing minimal bioavailability of the drug into the CNS, the BBB directly contributes towards the progression of the pathological conditions. This makes BBB a potential target for designing the drugs ${ }^{9}$ that can cross the BBB to treat CNS disorders. The progress of new chemical entity into clinically effective drugs is hindered by numerous drawbacks intrinsic to modelling the CNS in vitro and in vivo for instance, the post-mitotic nature of neurons (limited access to primary cultures), presence of an intricate tissue cyto-architecture, anatomical and molecular inter-species diversities, and constrained access to the human brain. Therefore, in order to study the drug transmigration across the BBB, simplified in vitro BBB models have been developed, including the monolayer models, co-culture models, dynamic models, stem cell-based and microfluidic models, to understand the dynamics and role of the BBB. The in vitro BBB models come with the shortcoming of not being able to be replicated in vivo conditions, therefore understanding the limitations of the in vitro BBB models would be critical for experimental design and data interpretation. ${ }^{1}$

\section{Fundamentals and composition of BBB}

The BBB consists of a monolayer of BMECs connected by much tighter junctions than peripheral vessels and forms a cellular membrane, which is known as the physical backbone of the BBB. ${ }^{8,10}$ The key characteristics of the BBB are its uniform thickness, absence of fenestrae, least pinocytotic activity, and negative surface charge. In the BBB composition, the capillary basement membrane, pericytes, astrocytes, and microglial cells forming the neurovascular unit support BMECs. The basement membrane is made of collagen and elastin structural proteins, fibronectin and laminin, which are specialized proteins and finally proteoglycans, which gives the structural specificity and membrane stability. Additionally, pericytes are the cellular constituents of microvessels, including capillaries and postcapillary venules, which cover $22-32 \%$ of the capillaries and share the same basement membrane, helping in various structural and non-structural tasks of the BBB. It synthesizes structural and signaling proteins and contributes to the BMECs proliferation, migration, and differentiation processes. ${ }^{10}$ The astrocytes are other important types of cells, contributing as a part of the neurovascular unit of CNS. Additionally, the presence of immunocompetent brain microglial cells is crucial that checks the local microenvironment with its motile extensions and are capable of changing phenotype according to the homeostatic disturbance in the CNS. ${ }^{11}$ The interactions of the BMECs with the basement membrane, glial cells (microglia and astrocytes), neurons, and perivascular pericytes lead to specific brain microvascular biology. Presence of matrix adhesion receptors and signaling proteins form an extensive and complex matrix that is essential for the maintenance of the BBB (Figure 1). ${ }^{10,12}$

\section{Molecular properties of BBB}

The molecular constituents of TJs, adherence junctions, and signaling pathways regulate the BMECs assembly. TJs are highly dynamic structures that are responsible for the selective permeability property of the $\mathrm{BBB}$ as the apical region of the endothelial cells are sealed together by TJs allowing limited paracellular permeability. Structurally, TJs are formed by the interaction of integral 


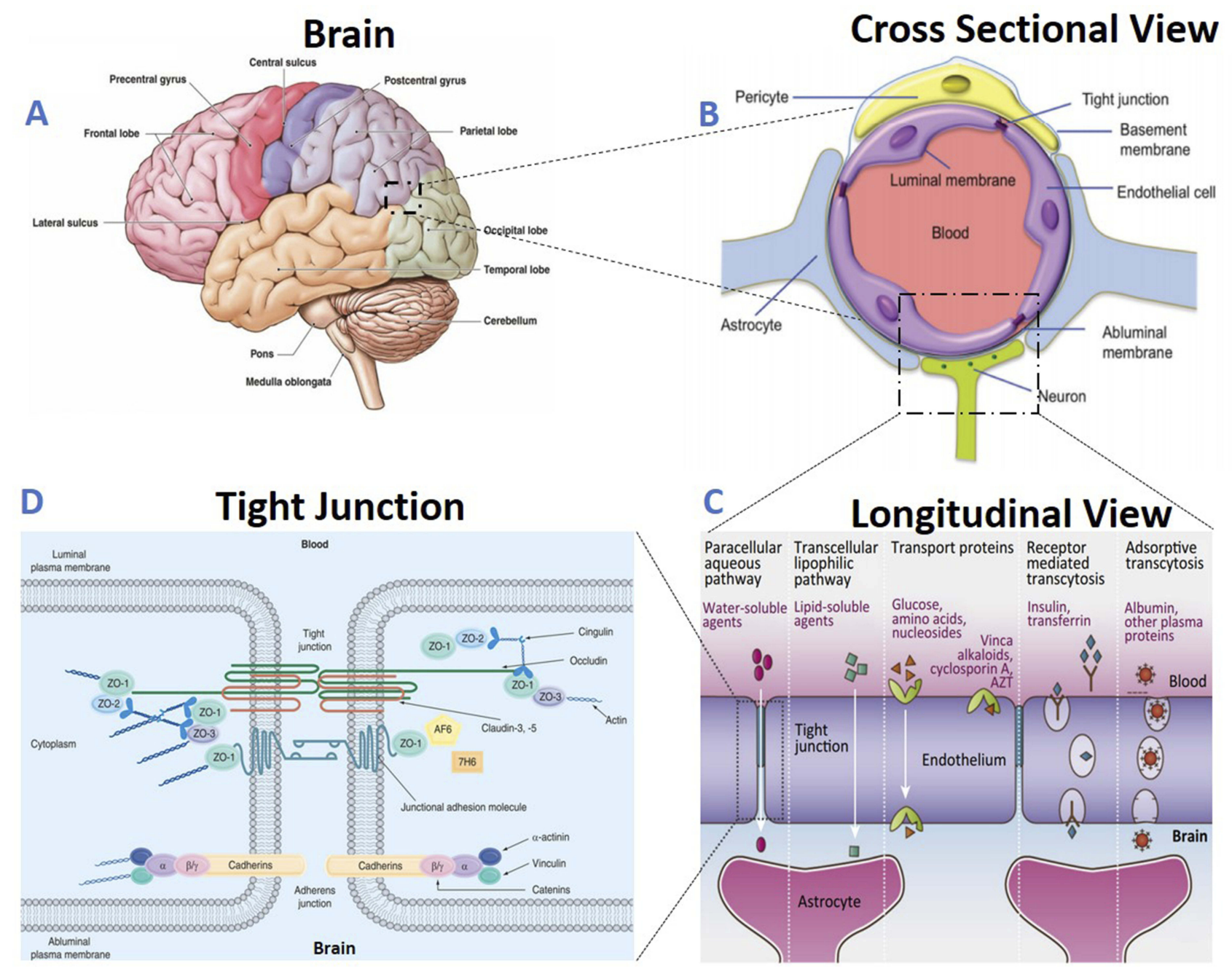

Figure I Structure and functionality of the Blood-Brain Barrier (BBB): (A) Brain structure- The brain has several barriers, including the BBB, the outer blood-cerebrospinal fluid (CSF)-brain barrier, and the blood-CSF barrier; (B) BBB structure- The BBB is formed by endothelial cells (ECs) that are in close association with astrocyte end feet and pericytes, forming a physical barrier; (C) BBB transport- Routes for molecular traffic across the BBB are shown. Some transporters are energy-dependent (Pglycoprotein, P-gP) and act as efflux transporters; (D) Tight junctions- Tight junctions are typically located on the apical region of ECs. The tight junctions form complex networks that result in multiple barriers that restrict the penetration of polar drugs into the brain. ${ }^{14,7}$

transmembrane proteins with the adjacent plasma membrane. ${ }^{13}$ Among these proteins, junction adhesion molecules (JAM), claudins and occludins (intermembrane) bind to cytoplasmic proteins (e.g. zonula occludens, cinguline), and are well-studied for their role in the TJs constituting the BBB. ${ }^{14,7}$ Along with contribution to the physical restriction property of the $\mathrm{BBB}$, TJs also provide functions such as control of gene expression, cell proliferation, and differentiation. Below the TJs, actin filaments (including cadherins and catenins) link together and form a belt of adherence junctions. ${ }^{6}$ These adherence junctions contribute to the barrier property along with additional roles such as promoting BMECs adhesions, contact inhibition throughout vascular growth, cell polarity and controlling paracellular permeability regulations. Dynamic interactions between TJs and adherence junctions, through signaling pathways, regulate the BBB permeability. These signaling pathways include mitogen-activated protein kinases, endothelial nitric oxide synthases, and G-proteins and interaction between these pathways regulate the paracellular route. The signal transduction includes signals from the interior of the cells to the TJs (facilitating the assembly and regulating the permeability) and signals from the TJs to the interior of the cell for modulating gene expression, proliferation and differentiation. ${ }^{8,13}$ In addition to the proteins with enzymatic activities, there are other specific proteins (i.e. drug efflux transporters (ATP-binding cassette (ABC),15 P-glycoprotein-Pgp, multidrug resistance proteins- MRPs, organic anion transporting polypeptides (OATPs), organic anion transporting 
polypeptides $)^{7,15}$ that work as the BBB transporters, responsible for the rapid efflux of xenobiotic/drugs from the CNS, and for the delivery of the essential nutrients and transmitters into the brain, resulting in the specific barrier functions of the BBB, important for protecting CNS against harmful xenobiotics. ${ }^{16-20} \mathrm{BBB}$ is complex, and transmigration of small/drugs molecules or drug-loaded nanoparticles still is a big challenge. ${ }^{21}$ The main drug transport mechanism for small/drug molecules for BBB permeation is attributed to passive diffusion, active transport, carrier-mediated/receptor-mediated and for larger peptide or proteins trans-endocytosis is the main transport mechanism (Figure 2). ${ }^{22,23}$ Other than physiological condition of basal membrane, physicochemical parameters of drugs (i.e. size, shape, charge molecular weight or lipophilicity) also effects the drug transport and need to be considered while designing the newer therapeutics. More details about the effect of these physicochemical parameters on BBB transport is covered in the review article by Teleanu et al $(2018)^{23}$ and research article by Surnar et al (2018). ${ }^{21}$

\section{In vitro BBB permeation measurement methods}

To expedite the process of brain research, and the development of novel drugs for numerous neurological diseases, different types of in vitro BBB models have been established. However, as none of these in vitro models entirely reproduces the in vivo conditions, there is no perfect in vitro BBB model. Therefore, it is important to carefully choose the in vitro $\mathrm{BBB}$ model according to the requirement of the study and interpretation of the data, efficiently. ${ }^{24-26}$ Here, we have summarized the commonly used in vitro BBB models, including the recently developed microfluidic BBB models, along with their advantages and disadvantages. Based on the simulation of shear stress, in vitro BBB models are classified into the static and dynamic BBB models.

\section{Static BBB models}

Static BBB models are commonly used, but they do not imitate the shear stress, which is

usually generated in vivo due to the blood flow. Static BBB models are further divided into monolayer and coculture models, based on type of cells involved in the BBB design.

\section{Monolayer BBB models}

A monolayer of endothelial cells grown in the Transwell insert is used as a simple in vitro BBB model (Figure 3A-i). The insert mimics the blood (luminal) side, whereas the well in which the insert fits, mimics the parenchymal (abluminal) side. The microporous membrane support $(0.2-0.4 \mu \mathrm{m})$ allows the exchange of small molecules and cell-secreted growth factors but prevents the migration of cells between the two compartments. To mimic the unique properties of BMECs, primary or low passage number cells are used for the BBB model preparation. This process is

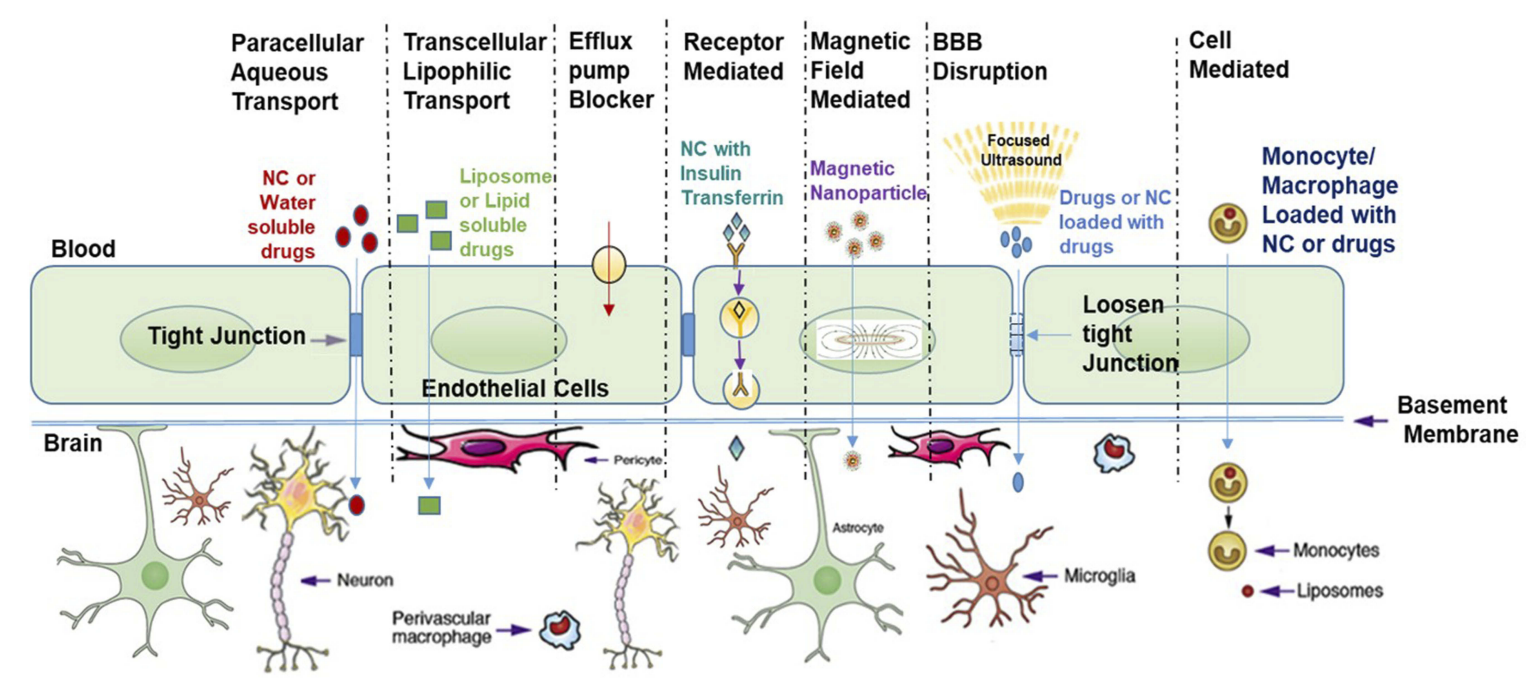

Figure 2 Schematic representation of mechanisms available for drugs transport across the BBB: Schematic shows the main mechanism behind the drugs or small molecule transport across the BBB ie receptor-mediated transcytosis; adsorptive transcytosis (passive transport), diffusion or active transport.

Notes: Reprinted from Adv Drug Deliv Rev, 103, Nair M, Jayant RD, Kaushik A, Sagar V., Getting into the brain: potential of nanotechnology in the management of NeuroAIDS, 202-217, Copyright 2016, with permission from Elsevier. ${ }^{14}$ 

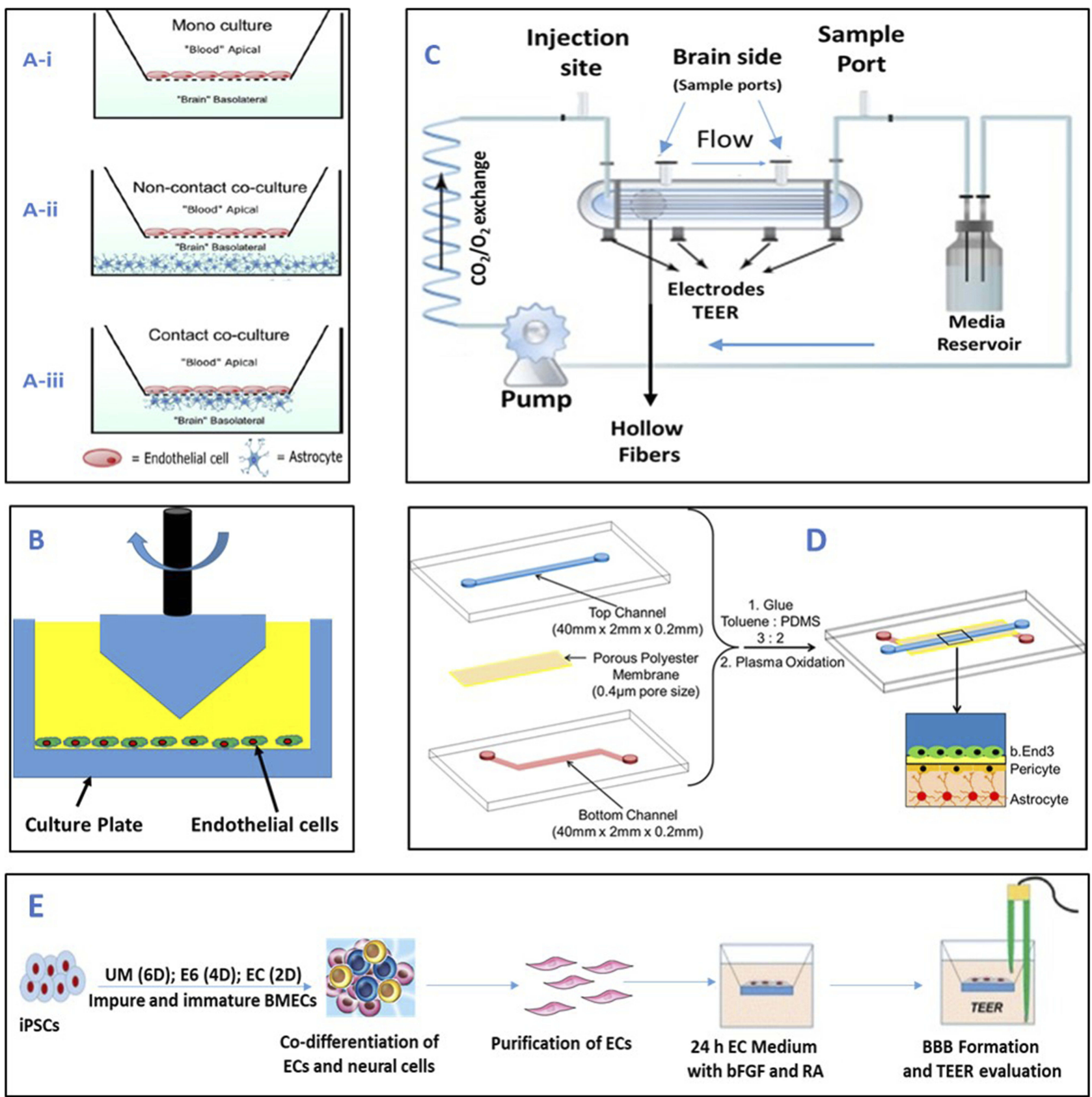

Figure 3 Schematic representation of different in vitro BBB models: (A) Configurations for in vitro static BBB Models using brain capillary endothelial cells (BCECs) (i) Monolayer models: are constructed using BCECs on the upper side of microporous semipermeable membrane (transwell), (ii) Non-contact co-culture: Astrocytes seeded at the bottom of the culture wells with BCECs; (iii) 2D co-culture contact models: endothelial cells are grown on porous cell culture inserts and co-cultured with primary astrocytes. Reprinted from J Pharm Sci, 105(2), Tornabene E, Brodin B, Stroke and drug delivery—in vitro models of the ischemic blood-brain barrier, Page Nos.398-405, Copyright 2016, with permission from Elsevier. ${ }^{83}$ (B) Cone and Plate viscometer apparatus. (C) Dynamic in vitro blood-brain barrier (DIV-BBB) model: The endothelial cells (ECs) are cultured inside the fibronectincoated surface of hollow fibers made up of polypropylene. This system allows co-culture because astrocytes can be cultured on the outer surface of the hollow fibers. (B) and (C) adapted from J Pharm Sci, I0I(4), Naik P, Cucullo L, In vitro blood-brain barrier models: current and perspective technologies, Page Nos. 1337-I354, Copyright 2012, with permission from Elsevier. ${ }^{50}$ (D) Microfluidic-based in vitro BBB models: layered PDMS channels sandwiching a polyester membrane and the organization of b.End3 endothelial cells, pericyte, and astrocytes in the co-culture model. Reprinted with permission from Wang JD, Khafagy E-S, Khanafer K, Takayama S, ElSayed MEH. Organization of endothelial cells, pericytes, and astrocytes into a 3D microfluidic in vitro model of the blood-brain barrier. Mol Pharm. 2016;13(3):895-906. Copyright @ 2016 American Chemical Society. ${ }^{84}$ (E) Stem cell-derived in-vitro BBB model: Undifferentiated iPSCs were differentiated simultaneously into ECs and neural cells, and then brain like ECs were purified on a selective matrix and co-cultured with astrocytes, the ECs exhibited a high TEER and formed networks of tight junctions.

Abbreviations: ACM-Astrocyte-conditioned medium; BMEC- Brain microvascular endothelial cell; and TEER- Transendothelial electric resistance; iPSCs- Induced pluripotent stem cells; UM- Unconditioned medium; E6-Essential medium; EC-Endothelial cell medium supplemented with bFGF (Basic fibroblast growth factor); RA-Retinoic acid.

challenging, due to isolation and culturing the primary BMECs as well as has a considerable risk of being contaminated by the mural cells. Also, low yield of primary BMECs after isolation is a shortcoming as the brain 
vasculature records only for $0.1 \%(\mathrm{v} / \mathrm{v})$ of the brain, therefore a large number of rodents are needed to isolate enough primary BMECs to continue with the cell culture studies. ${ }^{1,27}$ To overcome this limitation, larger species and nonhuman primates are utilized for the experiment to isolate significant amounts of BMECs, for further experiments. ${ }^{28}$ Human cells are used for the studies focusing human-specific transporters/receptors or immunological aspects, but insufficient because of ethical issues. To overcome this, many immortalized human cell lines, such as human cerebral microvascular endothelial cell line (hCMEC/D3) and immortalized human cerebral endothelial cells, have been produced, which are useful but have lower expression of some of the BBB specific transporters and enzymes, leading to decreased generation of a tight monolayer and thus, having inadequate barrier function. ${ }^{29}$ This inadequacy is counteracted by the addition of BBB modulating compounds like cAMP and glucocorticoids, which increase the endothelial monolayer tightness and stability. ${ }^{30,31}$ Monolayer models are employed in studying signaling pathways, transporter kinetics, binding affinity, and high-throughput screenings. However, the monolayer model is not ideal for BBB integrity studies, as it has only one type of cells (BMECs) and lacks to imitate the brain microenvironment in which cell to cell communication is essential between different cell CNS cell types. $^{32,33}$ Therefore, for the study of BBB integrity, more vivid and complex BBB model is required, such as co-culture and dynamic models.

\section{Co-culture BBB models}

In order to mimic the anatomic structure of $\mathrm{BBB}$ in vivo, BMECs are co-cultured with other CNS cells that directly contribute to the barrier properties of $\mathrm{BBB}$. Interaction between BMECs and other brain cells increases the expression of transporters, TJs in BMECs, and induces the cell polarity in BMECs, promoting a phenotype closely mimicking the BBB in vivo. In this co-culture model (BMECs with astrocytes or pericytes) fits (Figure 3A-ii). ${ }^{8,34-45}$ BMECs are seeded in the transwell insert, and astrocytes are grown either on the undersurface of the transwell insert or at the base of the well in which the insert. Since pericytes also have a key role in BBB regulation, a BMEC-astrocytepericyte co-culture model has also been developed, which is termed as a triple co-culture system (BMEC-pericyteastrocyte). Addition of pericytes enhances the quality of the co-culture model compared to the monolayer model. In this model, BMECs are plated in the transwell insert with astrocytes at the bottom of the well and pericytes on the underside of the insert (Figure 3A-iii). Although it lacks the direct cell-cell to communication between BMECs, astrocytes, and pericytes, this arrangement utilizes indirect cell-to-cell communication via secreted soluble factors, which promotes $\mathrm{BBB}$ regulation. BMEC-pericyte-astrocyte triple co-culture model is a more reliable in vitro $\mathrm{BBB}$ model due to the higher transendothelial electric resistance (TEER) value and lower permeability, which generates tighter BBB, ideal for permeability studies. ${ }^{27,46}$

\section{Dynamic BBB models}

In physiological conditions, the steady blood flow generates the shear stress, which regulates transporters and TJs expression, donating towards effective barrier function. Shear stress developed by blood flow increases ZO-1 expression and reduces permeability; therefore the dynamic BBB models, with shear stress, have been developed, which are of three major types, namely the coneplate, dynamic and microfluidic-based models. ${ }^{47,48}$

\section{Cone-plate BBB apparatus}

The cone-plate apparatus was used initially to construct shear force, in which a rotating cone produces shear force, and the angular velocity and the angle of the cone regulate the produced shear stress. The sheer stress then reaches the endothelial monolayer via the medium (Figure 3B), but it is not uniformly dispersed along the radius of the plates, and therefore the endothelial monolayer receives varying shear stress depending upon the location. This model does not include astrocytes and pericytes; therefore, it has a limited application, low reliability, and less significant to be used in the BBB studies. ${ }^{49,50}$

\section{Dynamic in vitro BBB model}

To incorporate both the components, i.e., shear stress and various cell types, microporous hollow fibers are used (Figure 3C). In this model, BMECs and astrocytes are implanted in the inner (luminal) and outer (abluminal) sides of the porous hollow fibers, respectively. ${ }^{1,50}$ The culture medium is pushed into the system through a variable-speed pump to produce shear stress equivalent to that of physiological conditions in vivo (5-23 dynes $\left./ \mathrm{cm}^{2}\right) .{ }^{51}$ To maintain the stable microenvironment, a gas-permeable tubing system was used for the exchange of $\mathrm{O}_{2}$ and $\mathrm{CO}_{2}$. This dynamic in vitro BBB model has been used to study the pathophysiology of various CNS diseases, including ischemia-reperfusion-induced injury and epilepsy. ${ }^{52,53}$ Recently, an updated model with hollow fibers with 
transmural microholes of $2-4 \mu \mathrm{m}$ has been developed to facilitate transmigration/trafficking studies. However, the dynamic BBB model has many disadvantages like, i) it does not allow direct visualization of the endothelial morphology in the luminal side; ii) the cell numbers $\left(>1 \times 10^{6}\right)$ required to build this model are relatively high, and iii) the time required to reach steady-state TEER is longer (912 days) compared to co-culture models (3-4 days). These shortcomings prevent the use of dynamic in vitro BBB model in large-scale screens. This model, however, is useful in lead compound validation/optimization in new drug research and development. ${ }^{53}$

\section{Microfluidic based BBB models}

Microfluidic device-based in vitro BBB models have been developed to overcome the shortcomings of dynamic BBB models. ${ }^{1,54,55}$ The microfluidic BBB ( $\mu$-BBB) comprises two perpendicularly crossing channels, allowing the dynamic flow to generate shear stress; a polycarbonate porous membrane placed over the intersection of the perpendicular channels, enabling the co-culture of BMECs and astrocytes (on the luminal and abluminal sides, respectively). The channels are $200 \mu \mathrm{m}$ tall, $2 \mathrm{~mm}$ (luminal) and $5 \mathrm{~mm}$ (abluminal) wide. It also contains multiple built-in $\mathrm{Ag} / \mathrm{AgCl}$ electrodes for facilitating the TEER measurement (Figure 3D). ${ }^{56}$ Pumps and a gas-permeable tubing system are used to generate shear stress and allow $\mathrm{O}_{2}-\mathrm{CO}_{2}$ exchange, respectively. This $\mu$-BBB model is further improved by replacing the oxidation-sensitive $\mathrm{AgCl}$ electrodes with inert platinum ones and reducing the cross-sectional area. These adjustments offer precise measurement of TEER and reduce the number of cells required. Additionally, the microfluidic-based BBB model with the microhole, have been designed to study the BBB permeability of drugs, which is composed of two horizontally aligned chambers connected by a microhole structure, but the shortcomings of the model is that i) it lacks cell-cell contact and, ii) it fails to replicate the dimensions of microvasculature in vivo. Therefore, currently, the development of a new version of the microfluidic device, i.e., a synthetic microvasculature model of the BBB (SyMBBB) is progressing. ${ }^{55}$ The SyM-BBB model contains two microchannels separated by microfabricated pillars, which mimic the porous membrane of the $\mu \mathrm{BBB}$ model. Endothelial cells were infused to the blood chamber via ports 1 and 2 , whereas astrocyte-conditioned medium or astrocytes were infused to the brain chamber from port 3 . The flow velocity of medium in these chambers regulates the shear stress.
This design mimics the in vivo microcirculatory system in a better way by including the diverging and converging bifurcations. In comparison to the dynamic BBB model, the microfluidic models are closer replicates of the in vivo BBB structure as they have thicker membrane $(<10 \mu \mathrm{m})$, which allows efficacious transmigration studies conditions. With the microfluidic model, nondestructive microscopy is possible because of the transparency of the materials, it takes relatively less time (3-4 days) to achieve steadystate TEER, and require only a modest amount of cells and are less challenging in terms of technical skills. ${ }^{54}$ Although the microfluidic systems have so many advantages, they have limitations too, i.e., 1) TEER value is not high enough $\left(250-300 \Omega \cdot \mathrm{cm}^{2}\right)$, ii) only incorporate two cell types, given that the membrane and pillars (hollow fibers in the dynamic in vitro BBB model) have only two sides. With the growth of research data, these microfluidic models may be used to aid neurovascular research and new drug development in the future due to its small size, short time to reach steady state TEER, low-to-moderate technical skill requirement, and low cost.

\section{Stem cell (iPSCs) based BBB models}

Pluripotent stem cells (PSCs), like embryonic stem (ES) cells and induced pluripotent stems cells (iPSCs) exhibit excellent properties that mimic physiological in vivo conditions. Stem cell-based in vitro systems have been extensively used for drug screening and regenerative therapies via differentiating cells from patient-derived pluripotent stem cells. For the in vitro BBB development, BMECs from human ES and iPSC by co-differentiation with neural cells along with retinoic acid have been explored comprehensively comparing to other available models (Figure 3E). The differentiated cells showed similar properties comprising barrier integrity with tissue derived BMECs. ${ }^{57}$ Yamanaka's contribution of deriving iPSCs from somatic cells and the neuronal differentiation procedure established by Zhang and co-workers on human ES, modelling neurological diseases in vitro using iPSCs had made a significant impact in the field of $\mathrm{BBB}$ and have been explored for the various neurological diseases like Parkinson's disease, Huntington's disease, Epilepsy, Down's syndrome, and autism disorders. ${ }^{58}$ Stem cell-derived BBB model provides a human, scalable, and reproducible source of cells, which can achieve good barrier properties like in vivo. Furthermore, the use of iPSCs provides an integrated model allowing the usage of isogenic co-cultures and consequently generate models similar to the complexity 
observed in vivo. The use of patient-specific in vitro models of the BBB generates unique aspects to detect how genetic disorders linked with neurological indications hamper the barrier function, thus, ultimately leading the researchers to identify novel therapeutic agents. Even though the iPSCs system offers great advantages, but the cell differential procedure depends upon various random and permanent insertion of transcription factors. Another major hurdle in diseases modelling using patient-derived iPSCs is the limited source of patient-derived iPSCs lines, shortage of matched-controls (e.g., parents, siblings) and a probable characteristic genetic drifting owing to the derivation and the maintenance of iPSC lines. Furthermore, the use of a genome integrating approach in the derivation method comprises a significant hurdle in translating such outcomes into iPSC-based cell therapies. Recent advances in gene editing techniques as CRISPR/Cas 9 provides exciting opportunities in diseases modelling using iPSCs. In addition, to tackle the problems mentioned above, various approaches have been developed, e.g. non-genomes integrating re-programming techniques like the usage of episomal vectors that allow cell-reprogramming bypasses, the insertion of genomic sequences have been developed. Although, these methods still have hindrances like epigenetic reprogramming and loss of patient-specific epigenetic signature. A significant problem of in vitro modelling is the absence of 3-dimensional structures related to cellular cytoarchitecture observed in vivo. A substitute to such matter is the portrayal of brain "organoids" offering a primitive multi-layer sphere. Although, these structures have several hindrances like the absence of an extracellular matrix scaffold (in vivo), absence of neuronal projections and tracts, and the presence of multi-cellular organoids integrative of glial cells. ${ }^{58}$

Zhang et al, produced an in vitro human BBB model by differentiating the human iPSC GM25256 cell line into brain endothelial cells. The model displayed various BBB features such as tight junction proteins (ZO-1, claudin-5, and occludin), endothelial markers (von Willebrand factor and Ulex), high transendothelial electrical resistance (TEER) value and c-GTPase activity. The TEER value significantly increased when co-cultured with primary rat astrocytes. RNAseq analysis verified the expression of all the important BBB related genes in the human iPSC derived endothelial cells in comparison to primary BMECs, comprising breast cancer resistant protein (BCRP) and P-glycoprotein (Pgp). Drug transport assays were also performed and in vitro results generated from this model could predict the in vivo BBB permeability of tested drugs in a successful manner. ${ }^{59}$ In another study by Lippmann et al showed that retinoic acid enhances the expression of vascular endothelial cadherin in differentiated ECs considerably before passage/purification, and in combination with hydrocortisone, it resulted in the higher TEER value in comparison to untreated cells. ${ }^{59}$ Researchers have also demonstrated the effect of co-culture with astrocytes and pericytes on purified BMECs to achieve better barrier and higher TEER measurements (6635 $\pm 315 \Omega \times \mathrm{cm}^{2}$; the highest reported value of TEER till now). ${ }^{60}$ Despite substantial progress, stem cell-derived in vitro BBB models demand cell surface marker enrichment or co-differentiation and purification actions generate a pure population of specialized brain endothelial cells still a big challenge.

iPSCs were also used to form neural progenitor-like EZ spheres, which can be differentiated into neurons and astrocytes, facilitating facile neural cell production. Canfield et al have developed an isogenic (each cell type obtained from the same patient) human BBB model containing iPSCs derived BMECs, astrocytes, and neurons. Furthermore, iPSC-derived BMECs co-culture with EZ-sphere-derived astrocytes and neurons generated in a sustained enhanced TEER compared to previously expressed models utilizing primary rat astrocytes and primary human NPCs as co-cultured neural cell sources. These models predicted that the generation of an isogenic model would help in applications for human BBB models and will serve as an excellent tool to evaluate human genetic disease on BBB, drug screening and toxicity evaluation for neurological disorders. ${ }^{61}$ The key features, advantages, and disadvantages of all the different type of in vitro BBB models are summarized in Table 1 and the comparison between different in vitro BBB models are also summarized in Table 2.

\section{BBB permeation prediction methods (in silico methods)}

The widely used in silico prediction of the BBB permeability is an inexpensive, less time consuming, and high throughput screening method for novel compounds in the drug discovery process. Although this method is based on several molecular descriptors or physicochemical characteristics of the molecule, it has its strengths and weaknesses. ${ }^{62-65}$ These computational models are typically based on the previous in vivo and in vitro experimental data. Therefore, for the predictive power of 
Table I Advantages and disadvantages of different in-vitro BBB models

\begin{tabular}{|c|c|c|}
\hline Model Type & Advantages & Disadvantage \\
\hline $\begin{array}{l}\text { Epithelial cells overexpressing } \\
\text { Transporters model }\end{array}$ & $\begin{array}{l}\text { - Cheap } \\
\text { - Easy to standardize }\end{array}$ & $\begin{array}{l}\text { - Differences between epithelial and endothelial } \\
\text { cells } \\
\text { - Non-physiologically high levels of transporter }\end{array}$ \\
\hline $\begin{array}{l}\text { Transwell monoculture model } \\
\text { - Cerebral endothelial cells on microporous } \\
\text { membranes }\end{array}$ & $\begin{array}{l}\text { - Uses brain endothelial cells } \\
\text { - Inexpensive }\end{array}$ & $\begin{array}{l}\text { - Effect of other cellular components of the neuro- } \\
\text { vascular unit (NVU-astrocytes, pericytes) is } \\
\text { neglected } \\
\text { - No shear stress }\end{array}$ \\
\hline $\begin{array}{l}\text { Co-cultures models } \\
\text { - Co-culture of cerebral microvascular } \\
\text { endothelial cells with astrocytes } \\
\text { - Co-culture models using pericytes } \\
\text { - Triple cell co-culture models (astrocytes, } \\
\text { endothelial and pericytes) } \\
\text { - Co-culture of brain endothelial cells with } \\
\text { neuronal precursors }\end{array}$ & $\begin{array}{l}\text { - Takes into account the influence of } \\
\text { other elements of the neurovascular } \\
\text { unit (NVU) }\end{array}$ & $\begin{array}{l}\text { - Relatively expensive and time-consuming } \\
\text { - No shear stress }\end{array}$ \\
\hline Dynamic in vitro (DIV) model & $\begin{array}{l}\text { - Mimics in-vivo situation possibility of } \\
\text { co-culture }\end{array}$ & $\begin{array}{l}\text { - Expensive } \\
\text { - No possibility to optically monitor the cells } \\
\text { - Special skills required to culture cells in these } \\
\text { conditions }\end{array}$ \\
\hline Microfluidic model & $\begin{array}{l}\text { - Mimics in-vivo situation possibility of } \\
\text { co-culture }\end{array}$ & - Not well-established models presently expensive \\
\hline iPSC (Pluripotent stem cells) based model & $\begin{array}{l}\text { - Generate models similar to the human } \\
\text { complexity observed in-vivo } \\
\text { - Very high TEER values }\end{array}$ & $\begin{array}{l}\text { - Differential procedure depends upon random and } \\
\text { permanent insertion of transcription factors, } \\
\text { - Complicated procedure with meagre yield } \\
\text { - Rigid removal of epigenetic markers related to } \\
\text { environmental exposure or age }\end{array}$ \\
\hline
\end{tabular}

Table 2 Comparison of different in vitro BBB models for drug transport

\begin{tabular}{|l|l|l|l|l|l|l|}
\hline Model Type & $\begin{array}{l}\text { Other brain } \\
\text { cell required }\end{array}$ & $\begin{array}{l}\text { Sheer } \\
\text { Stress } \\
\text { produced }\end{array}$ & $\begin{array}{l}\text { Time to } \\
\text { stable } \\
\text { TEER (d) }\end{array}$ & $\begin{array}{l}\text { Appropriate for } \\
\text { migration assay }\end{array}$ & Cost & $\begin{array}{l}\text { Technical } \\
\text { requisite }\end{array}$ \\
\hline Monolayer & No & No & $3-4 \mathrm{~d}$ & Yes & Low & Low \\
Co-culture & Yes & No & $3-4 \mathrm{~d}$ & Yes & Low to moderate \\
Cone-plate apparatus & No & Yes & $3-4 \mathrm{~d}$ & No & Low \\
Dynamic in vitro BBB & Yes & Yes & $9-12 \mathrm{~d}$ & No & High \\
Microfluidic based model & Yes & Yes & $3-4 \mathrm{~d}$ & Yes & High \\
iPSC based model & No & Yes & $>$ I Week & Yes & Moderate \\
High
\end{tabular}

estimations, the selection of datasets is a critical component. The assumption of passive diffusion of a compound as a major route of transport through the BBB is the base for the in silico predictions that do not consider various BBB transport pathways, e.g., nanoparticle-based transport/carrier-mediated, receptor-mediated, and active efflux or influx transport methods. ${ }^{66}$ Recently, cerebrospinal fluid (CSF) penetration is also considered in in silico model while analyzing the brain penetration of the molecule. ${ }^{67}$
To improve the predictive values of these computational models, novel sophisticated approaches have been developed. Table 3 shows different computational models to predict the BBB penetration property of newly designed or synthesized compounds. For brain penetration studies, brain-to-plasma ratios is measured and in silico extrapolations are based on the available $\log B \mathrm{~B}$ data, which represents the most readily available experimental data. ${ }^{65,68,69}$ In the training set, several molecular descriptors of the 
Table 3 In-silico models and their parameters used for predicting drug penetrability

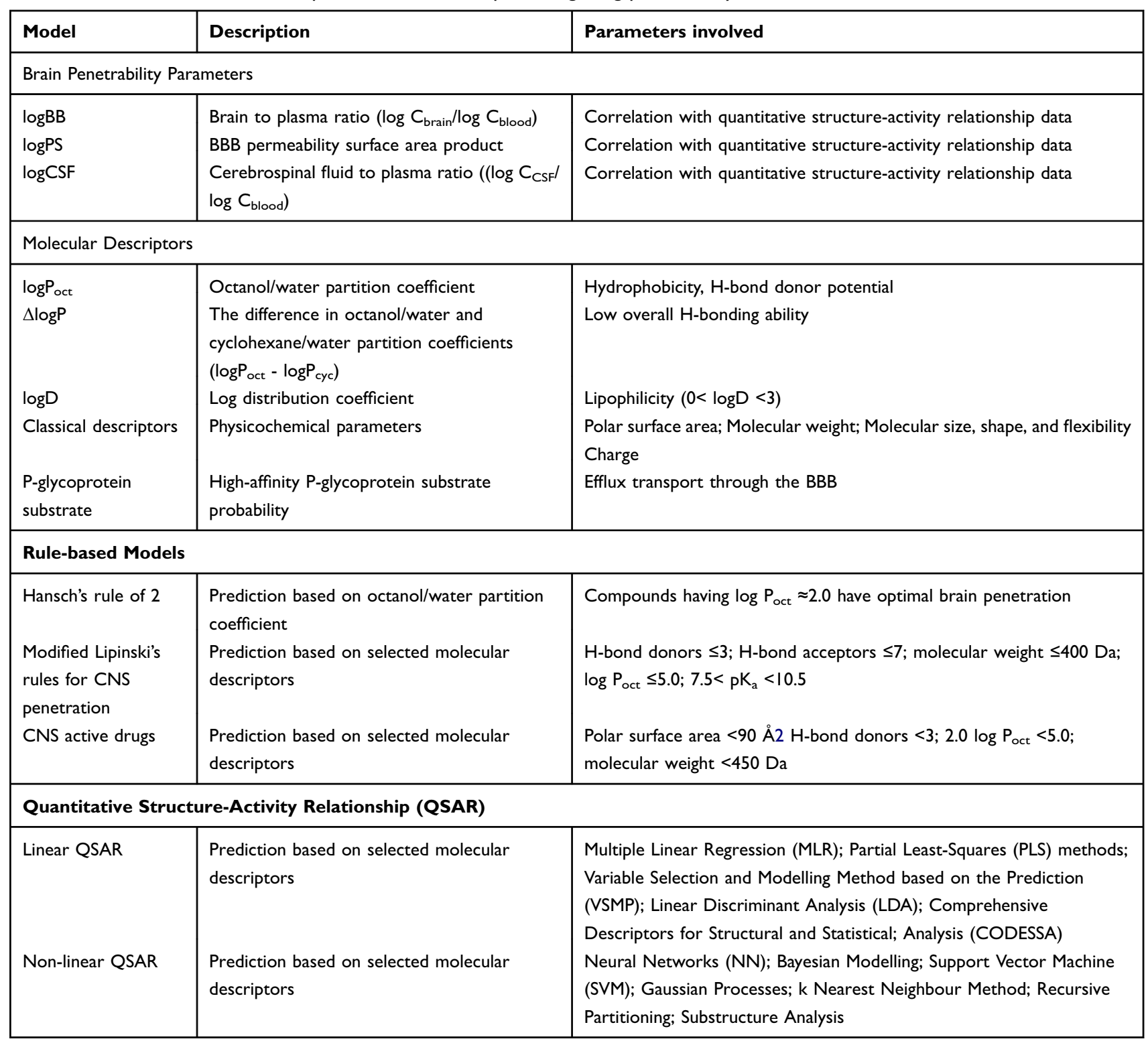

Abbreviations: $\mathrm{H}$, bond-hydrogen bond; $\log \mathrm{BB}$, brain to plasma ratio; $\log \mathrm{CSF}$, cerebrospinal fluid to plasma ratio; $\log \mathrm{D}$, $\log$ distribution coefficient; $\log \mathrm{P}$, log octanol/water partition coefficient; logPS, Blood-Brain-Barrier permeability surface area product; pKa, log of acidic dissociation constant.

compounds are calculated with known $\operatorname{logBB}$ values, which were experimentally determined. To derive the equation from the given relationship between $\log B \mathrm{~B}$ and the compound has computed descriptors, typically, regression methods used. Because of its physiological relevance, the permeability surface area product (PS value/logPS) would be an effective method of determining the BBB permeability for a specific molecule both in vivo and in vitro, compared with the currently more popular $\log \mathrm{BB} .{ }^{70,71}$ Unfortunately, the availability of $\log \mathrm{PS}$ is limited due to the complicated measurement of $\log P S$ than that of $\log B \mathrm{~B}$. Based on Lipinski's rule, molecules which have not more than $5 \mathrm{H}$-bond donors and not more than 10 H-bond acceptors, with an MW of $<500 \mathrm{Da}$ and an octanol/water partition coefficient log P under 5 can be the drug candidates. ${ }^{72}$ Molecules with these physicochemical characteristics have good aqueous solubility and intestinal permeability. Approximately $90 \%$ of the orally active drug molecules, which are under Phase II clinical trials have these characteristics. ${ }^{73}$ Guidelines for the properties of new molecules that can be used as potential CNS active drug have been proposed in recent past. ${ }^{73-76}$ The relationship between the experimental data, computationally available parameters of a new compound, and its blood-brain 
barrier penetration has been studied for long. Among different data sets, the octanol/water partitioning coefficient $\left(\log \mathrm{P}_{\text {oct }}\right)$ is one of the earliest predictive factors available for the BBB permeability. For the compounds with $\mathrm{MW}<400 \mathrm{Da}$, it is possible to predict the relationship of the capillary permeability coefficient $(\log \mathrm{PC})$ to the $\log \mathrm{P}_{\text {oct. }}{ }^{77}$ In 1988, when a linear correlation of antihistamines and $\Delta \log \mathrm{P}$ between the brain-to-blood ratio was established, the computational prediction of $\mathrm{BBB}$ penetration for these compounds began. ${ }^{78}$ The observation of the inverse relation between the hydrogen bonding activity of the compound and BBB permeability provided a theoretical concept for designing BBB-permeable drugs.

Calculations of $\log \mathrm{P}_{\text {oct }}$, -cyclohexane, and -dichloromethane systems were used to obtain the descriptors for general Linear Free Energy Response (LFER) equation. ${ }^{79}$ Calculation of physicochemical and biochemical properties of the compound would be useful to estimate the blood-to-brain distribution ratio. ${ }^{79}$ Universal quantitative scales of hydrogen-bond acidity and basicity of the solute have been standardized for the first time, and along with other descriptors, these descriptors have been used in equations to calculate, predict, analyze and correlate various solute properties. At the same time, these equations may be used for the analysis of physicochemical (LFERs) and biological properties of various compounds, such as quantitative structure-activity relationships (QSARs) set up for blood-brain distribution. Although these rule-based models can be used for the qualitative BBB permeability estimation using various physicochemical descriptors, these may not be useful to predict the active BBB transport through efflux pumps, carriers, and receptor-mediated transmigration. Overall, these in silico quantitative models as classification tools have more than $70 \%$ accuracy in predicting $\operatorname{logBB} .^{62,65}$ For the analysis of various molecular descriptors listed in Table 3, both linear and non-linear statistical methods would be used. These in silico models for predicting $\log \mathrm{BB}$ and $\log \mathrm{PS}$ are reliable and popular than in vitro and in vivo BBB models in the drug discovery process, as these methods are economical and faster. However, the size and quality of the training set play a key role in the accuracy of predicting the passive permeability. Similar to the recently developed model for P-glycoprotein substrate properties, if new models for the active transport mediated by carriers, receptors, and efflux pumps are developed, the predictive power of the in silico models will be tremendously increased. ${ }^{64,65,80}$

\section{The rationale for $\mathrm{BBB}$ model selection}

In vitro $\mathrm{BBB}$ models are extensively used for the initial stages of novel drug development, which includes lead identification, hit identification and target identification, optimization (Figure 4). Once a target (enzyme, receptors, etc.) is identified, high-throughput screening (HTS) is employed to identify probable drug contenders. At this phase, a large number of compounds need to be screened and thus, requires easy and fast in vitro BBB screening model. Selecting suitable in vitro models not only enables accurate interpretation of the data but also saves time and

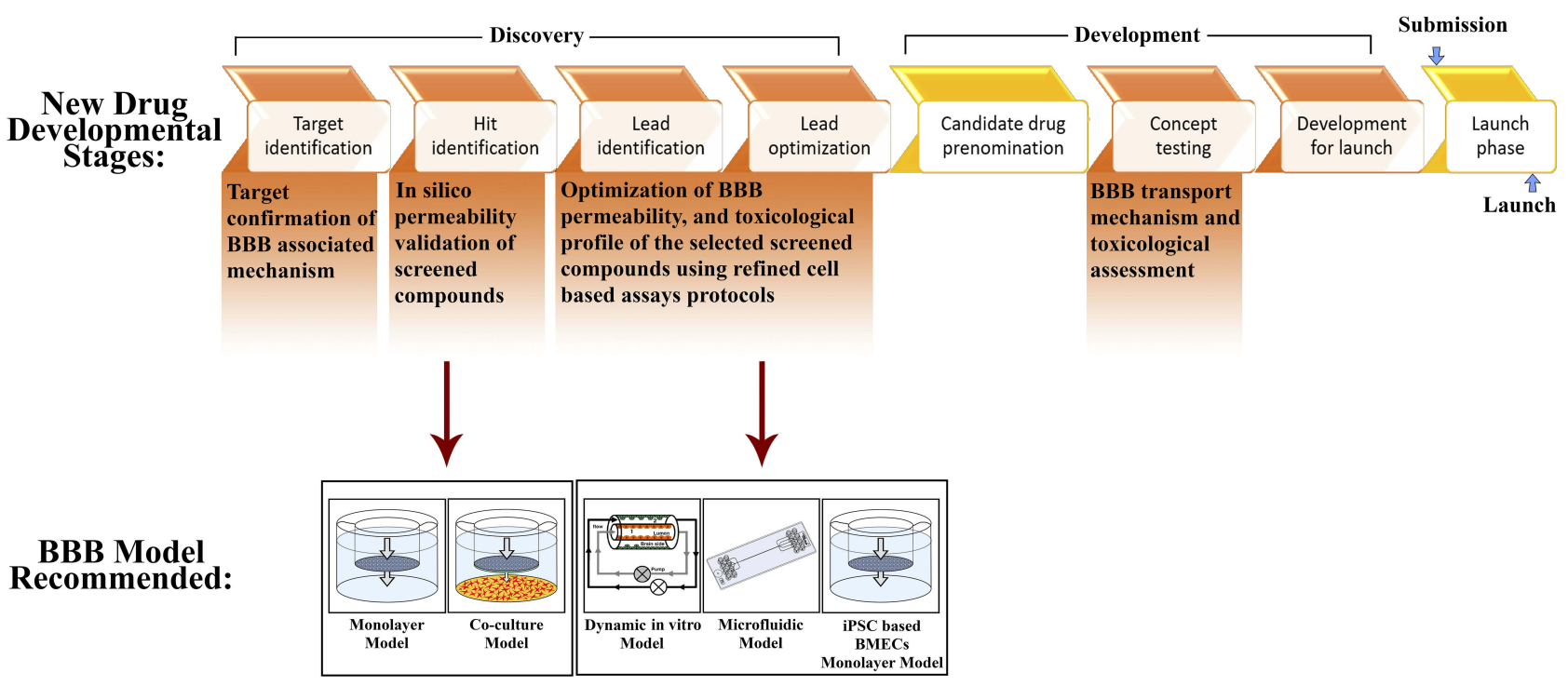

Figure 4 Applications of BBB models in drug discovery and development. 
money. The main criteria in the model selection are the purpose of study, in case of monolayer or co-culture models, which generally take 3-4 days to reach steady-state TEER value and are reasonably easy to construct, can be used. Previous studies have revealed that the use of different immortalized cell lines provides the best correlation between in vitro and in vivo data for permeability assays. The co-culture models and dynamic in vitro BBB models are the best models to study the drug permeability. ${ }^{1,25}$ Many multi-culture models (2D or 3D) are commercially available now, which considerably reduces the efforts and time, but increases the cost. For trafficking/migration studies, microfluidic BBB model is the right option due to the incorporation of shear stress component and ability to mimic in vivo conditions. ${ }^{1}$ To study signaling pathways/ transporter kinetics, or to quantify binding affinities, monolayer model is the best option due to its inherent simplicity. For the lead identification/optimization phase studies, validation, and structure-activity relationships (SAR) and toxicological profile, more sensitive in vitro models that replicate the majority of the in vivo conditions are required, i.e., iPSCs based model or static co-culture and dynamic models along with newly developed microfluidic-based BBB models can serve as an alternate choice. Primary human-derived cells are a better option than immortalized cell lines due to more closeness to biological properties of the BMECs in vivo. Authentication using human-derived primary cells is highly recommended for the generation of in vitro BBB model to evade species based difference that may lead to the failure of a product in the later development stages. Based on the above problems and suggestion, Figure 4 summarizes the selection of in vitro $\mathrm{BBB}$ models at different research and development stages for the therapeuticdrug development. ${ }^{28,62,68,81,82}$ The selection criteria of BBB models still a big puzzle for small molecule transport versus nanoparticle loaded drugs (Nanoformulation). Based on the available literature and newer development, iPSC based model (CNS organoids) may able to provide a better answer compared to traditional $2 \mathrm{D}$ or $3 \mathrm{D}$ cell-based models due to human-like pathophysiology and overcome the interspecies (rodent vs human) variability.

\section{Conclusion and future aspects}

In vitro models of the $\mathrm{BBB}$ have proven exceptionally valued for investigations of endo-thelial cell properties and mechanistic studies of drug transport via brain endothelial cells. The early effort of the pioneers has been followed up by a large community of investigators and has resulted in a range of in-vitro model configurations. In general, the cell culture models of the brain endothelium are believed to reflect the properties of the healthy $\mathrm{BBB}$. In vitro $\mathrm{BBB}$ models are essential to our understanding of the $\mathrm{BBB}$ functions in physiological and pathological situations and the research and development of novel drugs for different neurological conditions. Different in vitro BBB models have been established and used for a variety of permeation studies; no single model can imitate the in vivo conditions physiologically. Further, knowing the advantages and disadvantages of each of these models and rationale of selecting the appropriate model may allow the precise understanding of the data and elaborate the development of novel drugs for the treatment or management of neurological diseases. In summary, we attempt to provide an overview of regularly used, newly developed and advanced in vitro BBB models, equated their strengths and weaknesses and attempted to rationalize the model selection. Model selection parameters are critical for predicting drug transport because the disease in question may affect the barrier properties. A combinatorial approach of in vitro BBB models and invivo methods will be the key to the development of CNS therapeutics with improved pharmacokinetic properties and better BBB penetrability.

\section{Acknowledgments}

RDJ would like to acknowledge the financial support from NIH (R03DA044877); The Campbell Foundation (Florida, USA) and start-up funds from the Department of Pharmaceutical Sciences, School of Pharmacy, Texas Tech University Health Sciences Center (TTUHSC), TX, USA. VB would like to acknowledge the Department of Science and Technology, Ministry of Science and Technology, Government of India for the INSPIRE Faculty Award (IFA18-ENG266, DST/INSPIRE/04/2018/000991).

\section{Disclosure}

The authors report no conflicts of interest in this work.

\section{References}

1. He Y, Yao Y, Tsirka SE, Cao Y. Cell-culture models of the blood-brain barrier. Stroke. 2014;45(8):2514-2526. doi:10.1161/STROKEAHA.11 4.005427

2. Stern L, Gautier R II. Les Rapports Entre Le Liquide CéphaloRachidien Et Les éléments Nerveux De L'axe Cerebrospinal. Arch Int Physiol. 1922;17(4):391-448. doi:10.3109/13813452209146219 
3. Guillemin GJ, Brew BJ. Microglia, macrophages, perivascular macrophages, and pericytes: a review of function and identification. J Leukoc Biol. 2004;75(3):388-397. doi:10.1189/jlb.0303114

4. Cecchelli R, Berezowski V, Lundquist S, et al. Modelling of the blood-brain barrier in drug discovery and development. Nat Rev Drug Discov. 2007;6(8):650-661. doi:10.1038/nrd2368

5. Engelhardt B, Ransohoff RM. Capture, crawl, cross: the T cell code to breach the blood-brain barriers. Trends Immunol. 2012;33 (12):579-589. doi:10.1016/j.it.2012.07.004

6. Jouyban A, Soltani S. Blood brain barrier permeation. Toxic Drug Test. In: Bill Acree, Editor, Croatia: InTech. 2012;1:1-24.

7. Nair M, Jayant RD, Kaushik A, Sagar V. Getting into the brain: potential of nanotechnology in the management of NeuroAIDS. Adv Drug Deliv Rev. 2016;103:202-217. doi:10.1016/j.addr.2016. 02.008

8. Abbott NJ Physiology of the blood-brain barrier and its consequences for drug transport to the brain. Paper presented at: International Congress Series; 2005

9. Gajdács M. The concept of an ideal antibiotic: implications for drug design. Molecules. 2019;24(5):892. doi:10.3390/ molecules24050892

10. Cardoso FL, Brites D, Brito MA. Looking at the blood-brain barrier: molecular anatomy and possible investigation approaches. Brain Res Rev. 2010;64(2):328-363. doi:10.1016/j.brainresrev.2010.05.003

11. Prinz M, Mildner A. Microglia in the CNS: immigrants from another world. Glia. 2011;59(2):177-187. doi:10.1002/glia.21104

12. Aday S, Cecchelli R, Hallier-Vanuxeem D, Dehouck M, Ferreira L. Stem cell-based human blood-brain barrier models for drug discovery and delivery. Trends Biotechnol. 2016;34(5):382-393. doi:10. 1016/j.tibtech.2016.01.001

13. Ballabh P, Braun A, Nedergaard M. The blood-brain barrier: an overview: structure, regulation, and clinical implications. Neurobiol Dis. 2004;16(1):1-13. doi:10.1016/j.nbd.2003.12.016

14. Helms HC, Abbott NJ, Burek M, et al. In vitro models of the blood-brain barrier: an overview of commonly used brain endothelial cell culture models and guidelines for their use. $J$ Cereb Blood Flow Metab. 2016;36(5):862-890. doi:10.1177/ 0271678X16630991

15. Löscher W, Potschka H. Blood-brain barrier active efflux transporters: ATP-binding cassette gene family. NeuroRx. 2005;2(1):86-98. doi:10.1602/neurorx.2.1.86

16. Löscher W, Potschka H. Role of drug efflux transporters in the brain for drug disposition and treatment of brain diseases. Prog Neurobiol. 2005;76(1):22-76. doi:10.1016/j.pneurobio.2005.04.006

17. Kaushik A, Jayant RD, Bhardwaj V, Nair M. Personalized nanomedicine for CNS diseases. Drug Discov Today. 2018;23(5):1007-1015. doi:10.1016/j.drudis.2017.11.010

18. Kaushik A, Jayant RD, Nair M. Nanomedicine for neuroHIV/AIDS Management. London, UK: Future Medicine Ltd; 2018.

19. Kaushik A, Yndart A, Atluri V, et al. Magnetically guided noninvasive CRISPR-Cas9/gRNA delivery across blood-brain barrier to eradicate latent HIV-1 infection. Sci Rep. 2019;9(1):3928. doi:10.1038/s41598-019-40222-4

20. Lee M, Jayant R. Penetration of the blood-brain barrier by peripheral neuropeptides: new approaches to enhancing transport and endogenous expression. Cell Tissue Res. 2019;375(1):287-293. doi:10.1007/ s00441-018-2959-y

21. Surnar B, Basu U, Banik B, et al. Nanotechnology-mediated crossing of two impermeable membranes to modulate the stars of the neurovascular unit for neuroprotection. Proc Natl Acad Sci. 2018;115(52) E12333-E12342. doi:10.1073/pnas.1816429115

22. Alavijeh MS, Chishty M, Qaiser MZ, Palmer AM. Drug metabolism and pharmacokinetics, the blood-brain barrier, and central nervous system drug discovery. NeuroRx. 2005;2(4):554-571. doi:10.1602/ neurorx.2.4.554
23. Teleanu D, Chircov C, Grumezescu A, Volceanov A, Teleanu R. Bloodbrain delivery methods using nanotechnology. Pharmaceutics. 2018;10 (4):269. doi:10.3390/pharmaceutics 10040269

24. Czupalla CJ, Liebner S, Devraj K. In vitro models of the blood-brain barrier. In: Milner R. Editor. Cerebral Angiogenesis: Methods and Protocols; NY: Humana Press. 2014;1135:415-437.

25. Garberg P, Ball M, Borg N, et al. In vitro models for the blood-brain barrier. Toxicol in Vitro. 2005;19(3):299-334. doi:10.1016/j.tiv.2004. 06.011

26. Wilhelm I, Fazakas C, Krizbai IA. In vitro models of the blood-brain barrier. Acta Neurobiol Exp (Wars). 2011;71(1):113-128.

27. Nakagawa S, Deli MA, Kawaguchi H, et al. A new blood-brain barrier model using primary rat brain endothelial cells, pericytes and astrocytes. Neurochem Int. 2009;54(3):253-263. doi:10.1016/j. neuint.2008.12.002

28. Lippmann ES, Al-Ahmad A, Palecek SP, Shusta EV. Modeling the blood-brain barrier using stem cell sources. Fluids Barriers CNS. 2013;10(1):2. doi:10.1186/2045-8118-10-2

29. Daniels BP, Cruz-Orengo L, Pasieka TJ, et al. Immortalized human cerebral microvascular endothelial cells maintain the properties of primary cells in an in vitro model of immune migration across the blood brain barrier. J Neurosci Methods. 2013;212(1):173-179. doi:10.1016/j.jneumeth.2012.10.001

30. Franke H, Galla H-J, Beuckmann CT. An improved low-permeability in vitro-model of the blood-brain barrier: transport studies on retinoids, sucrose, haloperidol, caffeine and mannitol. Brain Res. 1999;818(1):65-71. doi:10.1016/s0006-8993(98)01282-7

31. Hurst R, Fritz I. Properties of an immortalised vascular endothelial/ glioma cell co-culture model of the blood-brain barrier. $J$ Cell Physiol. 1996;167(1):81-88. doi:10.1002/(SICI)1097-4652(199604) 167:1<81::AID-JCP9>3.0.CO;2-8

32. Hori S, Ohtsuki S, Hosoya K, Nakashima E, Terasaki T. A pericytederived angiopoietin-1 multimeric complex induces occludin gene expression in brain capillary endothelial cells through Tie-2 activation in vitro. $J$ Neurochem. 2004;89(2):503-513. doi:10.1111/j.14714159.2004.02343.x

33. Toimela T, Mäenpää H, Mannerström M, Tähti H. Development of an in vitro blood-brain barrier model-cytotoxicity of mercury and aluminum. Toxicol Appl Pharmacol. 2004;195(1):73-82. doi:10.10 16/j.taap.2003.11.002

34. Armulik A, Genové G, Mäe M, et al. Pericytes regulate the bloodbrain barrier. Nature. 2010;468(7323):557-561. doi:10.1038/nature09 522

35. Daneman R, Zhou L, Kebede AA, Barres BA. Pericytes are required for blood-brain barrier integrity during embryogenesis. Nature. 2010;468(7323):562-566. doi:10.1038/nature09513

36. Atluri VSR, Jayant RD, Pilakka-Kanthikeel S, et al. Development of TIMP1 magnetic nanoformulation for regulation of synaptic plasticity in HIV-1 infection. Int J Nanomedicine. 2016;11:4287. doi:10. 2147/IJN.S108329

37. Jayant R. Layer-by-Layer (LbL) assembly of anti HIV drug for sustained release to brain using magnetic nanoparticle. Paper presented at: Journal of Neuroimmune Pharmacology; 2014.

38. Jayant R, Nair M. Nanotechnology for the treatment of neuroAIDS. $J$ Nanomed Res. 2016;3(1):00047. doi:10.15406/jnmr.2016.03.00047

39. Jayant R, Nair M. Role of biosensing technology for neuroAIDS management. J Biosens Bioelectron. 2016;7(1).

40. Jayant RD, Madhavan N Materials and methods for sustained release of active compounds. US Patent App. 15/082,611. 2016.

41. Kaushik A, Jayant RD, Nair M. Advancements in nano-enabled therapeutics for neuroHIv management. Int $J$ Nanomedicine. 2016;11:4317. doi:10.2147/IJN.S109943

42. Tomitaka A, Arami H, Raymond A, et al. Development of magnetoplasmonic nanoparticles for multimodal image-guided therapy to the brain. Nanoscale. 2017;9(2):764-773. doi:10.1039/c6nr07520g 
43. Nair M, Guduru R, Liang P, Hong J, Sagar V, Khizroev S. Externally controlled on-demand release of anti-HIV drug using magneto-electric nanoparticles as carriers. Nat Commun. 2013;4:1707. doi:10.1038/ncomms2717

44. Pilakka-Kanthikeel S, Atluri VSR, Sagar V, Saxena SK, Nair M. Targeted brain derived neurotropic factors (BDNF) delivery across the blood-brain barrier for neuro-protection using magnetic nano carriers: an in-vitro study. PLoS One. 2013;8(4):e62241. doi:10. 1371/journal.pone.0062241

45. Ding H, Sagar V, Agudelo M, et al. Enhanced blood-brain barrier transmigration using a novel transferrin embedded fluorescent magneto-liposome nanoformulation. Nanotechnology. 2014;25(5):055 101. doi:10.1088/0957-4484/25/5/055101

46. Nakagawa S, Deli MA, Nakao S, et al. Pericytes from brain microvessels strengthen the barrier integrity in primary cultures of rat brain endothelial cells. Cell Mol Neurobiol. 2007;27(6):687-694. doi:10.1007/s10571-007-9195-4

47. Siddharthan V, Kim YV, Liu S, Kim KS. Human astrocytes/astrocyteconditioned medium and shear stress enhance the barrier properties of human brain microvascular endothelial cells. Brain Res. 2007;1147:39-50. doi:10.1016/j.brainres.2007.02.029

48. Tarbell JM. Shear stress and the endothelial transport barrier. Cardiovasc Res. 2010;87(2):320-330. doi:10.1093/cvr/cvq146

49. Bussolari SR, Dewey CF Jr, Gimbrone MA Jr. Apparatus for subjecting living cells to fluid shear stress. Rev Sci Instrum. 1982;53 (12): 1851-1854. doi:10.1063/1.1136909

50. Naik P, Cucullo L. In vitro blood-brain barrier models: current and perspective technologies. J Pharm Sci. 2012;101(4):1337-1354. doi:10.1002/jps. 23022

51. Koutsiaris AG, Tachmitzi SV, Batis N, et al. Volume flow and wall shear stress quantification in the human conjunctival capillaries and post-capillary venules in vivo. Biorheology. 2007;44(56):375-386.

52. Cucullo L, Hossain M, Rapp E, Manders T, Marchi N, Janigro D. Development of a humanized in vitro blood-brain barrier model to screen for brain penetration of antiepileptic drugs. Epilepsia. 2007;48 (3):505-516. doi:10.1111/j.1528-1167.2006.00960.x

53. Cucullo L, Marchi N, Hossain M, Janigro D. A dynamic in vitro BBB model for the study of immune cell trafficking into the central nervous system. J Cereb Blood Flow Metab. 2011;31(2):767-777. doi:10.1038/jcbfm.2010.162

54. Booth R, Kim H. Characterization of a microfluidic in vitro model of the blood-brain barrier ( $\mu$ BBB). Lab Chip. 2012;12(10):1784-1792. doi: $10.1039 / \mathrm{c} 2 \mathrm{lc} 40094 \mathrm{~d}$

55. Prabhakarpandian B, Shen M-C, Nichols JB, et al. SyM-BBB: a microfluidic blood brain barrier model. Lab Chip. 2013;13(6):10931101. doi:10.1039/c2lc41208j

56. Booth R, Kim H A multi-layered microfluidic device for in vitro bloodbrain barrier permeability studies. Paper presented at: International Conference on Miniaturized Systems for Chemistry and Life Sciences; 2011.

57. Kokubu Y, Yamaguchi T, Kawabata K. In vitro model of cerebral ischemia by using brain microvascular endothelial cells derived from human induced pluripotent stem cells. Biochem Biophys Res Commun. 2017;486(2):577-583. doi:10.1016/j.bbrc.2017.03.092

58. Page S, Patel R, Raut S, Al-Ahmad A. Neurological diseases at the blood-brain barrier: stemming new scientific paradigms using patientderived induced pluripotent cells. Biochim Biophys Acta Mol Basis Dis. 2018. doi:10.1016/j.bbadis.2018.12.009

59. Li Y, Sun X, Liu H, et al. Development of human in vitro brain-blood barrier model from induced pluripotent stem cell-derived endothelial cells to predict the in vivo permeability of drugs. Neurosci Bull. 2019:1-15. doi:10.1007/s12264-019-00384-7
60. Hollmann EK, Bailey AK, Potharazu AV, Neely MD, Bowman AB, Lippmann ES. Accelerated differentiation of human induced pluripotent stem cells to blood-brain barrier endothelial cells. Fluids Barriers CNS. 2017;14(1):9. doi:10.1186/s12987-0170059-0

61. Canfield SG, Stebbins MJ, Morales BS, et al. An isogenic bloodbrain barrier model comprising brain endothelial cells, astrocytes, and neurons derived from human induced pluripotent stem cells. J Neurochem. 2017;140(6):874-888. doi:10.1111/jnc.13923

62. Vastag M, Keseru GM. Current in vitro and in silico models of bloodbrain barrier penetration: a practical view. Curr Opin Drug Discov Devel. 2009;12(1):115-124.

63. Abbott NJ. Prediction of blood-brain barrier permeation in drug discovery from in vivo, in vitro and in silico models. Drug Discov Today Technol. 2004;1(4):407-416. doi:10.1016/j.ddtec.2004.11.014

64. Goodwin JT, Clark DE. In silico predictions of blood-brain barrier penetration: considerations to "Keep in mind". J Pharmacol Exp Ther. 2005;315(2):477-483. doi:10.1124/jpet.104.075705

65. Mensch J, Oyarzabal J, Mackie C, Augustijns P. In vivo, in vitro and in silico methods for small molecule transfer across the BBB. $J$ Pharm Sci. 2009;98(12):4429-4468. doi:10.1002/jps.21745

66. Deli MA. Drug Transport and the Blood-brain Barrier. Solubility, Delivery, and ADME Problems of Drugs and Drug-Candidates. Washington: Bentham Science Publ Ltd; 2011:144-165.

67. Bendels S, Kansy M, Wagner B, Huwyler J. In silico prediction of brain and CSF permeation of small molecules using PLS regression models. Eur J Med Chem. 2008;43(8):1581-1592. doi:10.1016/j. ejmech.2007.11.011

68. Garg P, Verma J, Roy N. In silico modeling for blood—brain barrier permeability predictions. In: Ehrhardt C, Kim KJ, Editors. Drug Absorption Studies. Springer; 2008;VII:510-556.

69. Konovalov DA, Coomans D, Deconinck E, Vander Heyden Y. Benchmarking of QSAR models for blood-brain barrier permeation. $J$ Chem Inf Model. 2007;47(4):1648-1656. doi:10.1021/ci700100f

70. Liu X, Tu M, Kelly RS, Chen C, Smith BJ. Development of a computational approach to predict blood-brain barrier permeability. Drug Metab Dispos. 2004;32(1):132-139. doi:10.1124/dmd.32.1.132

71. Abraham MH. The factors that influence permeation across the blood-brain barrier. Eur J Med Chem. 2004;39(3):235-240. doi:10.1016/j.ejmech.2003.12.004

72. Lipinski CA, Lombardo F, Dominy BW, Feeney PJ. Experimental and computational approaches to estimate solubility and permeability in drug discovery and development settings. Adv Drug Deliv Rev. 1997;23(1-3):3-25. doi:10.1016/S0169-409X(96)00423-1

73. Lipinski CA. Lead-and drug-like compounds: the rule-of-five revolution. Drug Discov Today Technol. 2004;1(4):337-341. doi:10.1016/j. ddtec.2004.11.007

74. Glave W, Hansch C. Relationship between lipophilic character and anesthetic activity. J Pharm Sci. 1972;61(4):589-591. doi:10.1002/ jps. 2600610420

75. Pajouhesh H, Lenz GR. Medicinal chemical properties of successful central nervous system drugs. NeuroRx. 2005;2(4):541-553. doi:10.1602/neurorx.2.4.541

76. Hitchcock SA. Blood-brain barrier permeability considerations for CNS-targeted compound library design. Curr Opin Chem Biol. 2008;12(3):318-323. doi:10.1016/j.cbpa.2008.03.019

77. Levin VA, Dolginow D, Landahl HD, Yorke C, Csejtey J. Relationship of octanol/water partition coefficient and molecular weight to cellular permeability and partitioning in S49 lymphoma cells. Pharm Res. 1984;1(6):259-266. doi:10.1023/A:1016393902123

78. Young RC, Mitchell RC, Brown TH, et al. Development of a new physicochemical model for brain penetration and its application to the design of centrally acting $\mathrm{H} 2$ receptor histamine antagonists. $J$ Med Chem. 1988;31(3):656-671. doi:10.1021/jm00398a028 
79. Abraham MH, Takács-Novák K, Mitchell RC. On the partition of ampholytes: application to blood-brain distribution. J Pharm Sci. 1997;86(3):310-315. doi:10.1021/js960328j

80. Liu X, Chen C, Smith BJ. Progress in brain penetration evaluation in drug discovery and development. J Pharmacol Exp Ther. 2008;325 (2):349-356. doi:10.1124/jpet.107.130294

81. Sakolish CM, Esch MB, Hickman JJ, Shuler ML, Mahler GJ. Modeling barrier tissues in vitro: methods, achievements, and challenges. EBioMedicine. 2016;5:30-39. doi:10.1016/j.ebiom.2016.02. 023
82. Veszelka S, Kittel Á, Deli MA. Tools of Modelling Blood-brain Barrier Penetrability. Solubility, Delivery and ADME Problems of Drugs and Drug Candidates. Washington: Bentham Science; 2011:166-188.

83. Tornabene E, Brodin B. Stroke and drug delivery-in vitro models of the ischemic blood-brain barrier. J Pharm Sci. 2016;105(2):398-405. doi:10.1016/j.xphs.2015.11.041

84. Wang JD, Khafagy E-S, Khanafer K, Takayama S, ElSayed MEH. Organization of endothelial cells, pericytes, and astrocytes into a 3D microfluidic in vitro model of the blood-brain barrier. Mol Pharm. 2016;13(3):895-906. doi:10.1021/acs.molpharmaceut.5b00805

\section{Publish your work in this journal}

Drug Design, Development and Therapy is an international, peerreviewed open-access journal that spans the spectrum of drug design and development through to clinical applications. Clinical outcomes, patient safety, and programs for the development and effective, safe, and sustained use of medicines are a feature of the journal, which has also been accepted for indexing on PubMed Central. The manuscript management system is completely online and includes a very quick and fair peer-review system, which is all easy to use. Visit http://www. dovepress.com/testimonials.php to read real quotes from published authors. 